\title{
A Mathematical Modelling Technique for the Analysis of the Dynamics of a Simple Continuous EDA
}

\author{
Bo Yuan, Student Member, IEEE, and Marcus Gallagher, Member, IEEE
}

\begin{abstract}
This paper presents some initial attempts to mathematically model the dynamics of a continuous Estimation of Distribution Algorithm (EDA) based on Gaussian distributions. Case studies are conducted on both unimodal and multimodal problems to highlight the effectiveness of the proposed technique and explore some fundamental issues of the EDA. With some general assumptions, we can show that, for one-dimensional unimodal problems and with the $(\mu, \lambda)$ scheme: (1). The convergence behaviour of the EDA is independent of the test function except its general shape; (2). When starting far away from the global optimum, the EDA may get stuck; (3). Given a certain selection pressure, there is a unique parameter value that could help the EDA achieve desirable performance; for one-dimensional multimodal problems: (1). The EDA could get stuck with the $(\mu, \lambda)$ scheme; (2). The EDA will never get stuck with the $(\mu+\lambda)$ scheme.
\end{abstract}

\section{INTRODUCTION}

Estimation of Distribution Algorithms (EDAs) [8] refer to a relatively new paradigm of Evolutionary Algorithms (EAs), which is based on statistical Machine Learning techniques instead of conventional genetic operators such as crossover and mutation. The major advantage of EDAs is that they can explicitly build a probability density function based on the distribution of promising individuals and utilize the dependence information to conduct optimization efficiently, which is particular important upon the presence of strong epistasis among variables.

Usually, EAs are evaluated on the empirical basis while only limited progresses have been made in the theoretical aspect, especially compared to the large number of algorithms in the literature[1]. This is mainly due to their complex dynamics and massive parallel behaviour as well as the lack of appropriate modelling techniques.

In traditional EAs, new individuals are generated by directly manipulating current individuals through various stochastic genetic operators. Consequently, it may be not straightforward to precisely estimate the new population and thus predict the detailed evolution process in each generation. Fortunately, this issue may be less challenging in EDAs because the searching process is solely driven by a highlevel statistical model and all individuals are generated by sampling from this model. In order to describe the behaviour

Bo Yuan is with the School of Information Technology and Electrical Engineering, The University of Queensland, Brisbane, QLD 4072, Australia (phone: +61-7-3365-1636; email: boyuan@itee.uq.edu.au)

Marcus Gallagher is with the School of Information Technology and Electrical Engineering, The University of Queensland, Brisbane, QLD 4072 , Australia (phone: +61-7-3365-6197; email: marcusg@itee.uq.edu.au) of an EDA, only the model needs to be estimated, which can often be fully specified by a few parameters. This clarity in the mechanism of EDAs makes it possible to conduct more detailed theoretical analysis[2, 4, 7, 9-11, 13].

In this paper, a general technique is proposed to model the behaviour of a continuous EDA based on Gaussian distributions and Truncation Selection, which is aimed at precisely predicting the Gaussian model in each generation specified by the mean and standard deviation parameters. The importance of this technique is that it can be used to theoretically investigate some fundamental issues such as the influence of various algorithm factors and the problem structure.

As to the previous related work that we are aware of, González et. al and Grahl et. al present some theoretical results for the $\mathrm{UMDA}_{\mathrm{c}}$ algorithm, which is equal to the EDA used in this paper in one dimensional spaces [5, 6]. However, our work is different in several aspects. For example, we consider not only unimodal but also multimodal problems on which some interesting dynamics could be observed. Furthermore, in addition to the $(\mu, \lambda)$ scheme, the more complex $(\mu+\lambda)$ scheme is also taken into account (i.e., in both cases $\mu=\lambda$ ), which has not been attempted before.

\section{ESTIMATION OF DISTRIBUTION ALGORITHMS}

TABLE I

THE GENERAL FRAMEWORK OF THE GAUSSIAN EDA

Initialize and evaluate the starting population $\mathrm{P}$

While stopping criteria not met

Select the top individuals $\mathrm{P}^{\text {sel }}$

Fit a Gaussian model $\theta$ to $\mathrm{P}^{\text {sel }}$

Sample a set of individuals $\mathrm{P}^{\prime}$ from $\theta$

Evaluate new individuals in $\mathrm{P}^{\prime}$

Use $\mathrm{P}^{\prime}$ as the new population

or

Select the top individuals from P U P'

End While

The general mechanism of EDAs is an iterative process of evolving a statistical model such as Gaussian models, Gaussian Mixture models and Gaussian Networks, which specifies the distribution of the promising individuals in the current population. The major motivation is that, with the guidance of the statistical model, EDAs are expected to be able to explicitly capture the dependence relationship among 
problem parameters and may achieve faster convergence speed when complex dependence relationship does exist in the problem of interest.

In the continuous EDA used in this paper (Table 1), a portion of top individuals in the current population $\mathrm{P}$ are selected using Truncation Selection at each generation and a multivariate Gaussian model $\theta$ with full covariance structure is then built using the maximum likelihood estimate. All new individuals $\mathrm{P}^{\prime}$ are generated by sampling from $\theta$ and the new population is created through either the $(\mu, \lambda)$ scheme (i.e., replace all old individuals) or the $(\mu+\lambda)$ scheme (i.e., choose the top individuals from the union of old and new individuals). Note that $\mathrm{P}$ and $\mathrm{P}^{\prime}$ are of the same size in this framework (i.e., $\mu=\lambda$ ).

\section{MODELLING UNIMODAL PROBLEMS}

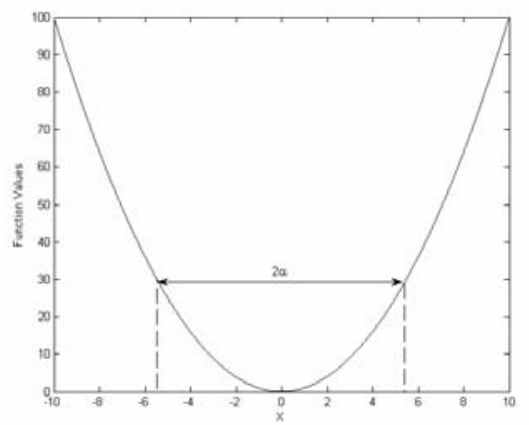

Fig. 1. A typical one-dimensional unimodal problem

The 1D unimodal problems considered in this section have the general structure like the quadratic function $y=x^{2}$ as shown in Figure 1. The only assumption is that each problem is symmetric with regard to its global minimum, which is placed at the origin without loss of generality (i.e., there are no upper and lower boundaries). For the EDA, an infinitely large population size is assumed so that new individuals have exactly the same statistics as the Gaussian model from which they are sampled. However, as will be shown in the experiments, a moderate population size is often sufficient to produce a close match between empirical and theoretical results. Furthermore, it is assumed that the initial population is generated from a Gaussian $\left(\mu_{0}, \delta_{0}\right)$. During evolution, the Gaussian model will be evolved towards the origin with changing mean and standard deviation values. A demo of the dynamics of the EDA is shown in Figure 2.

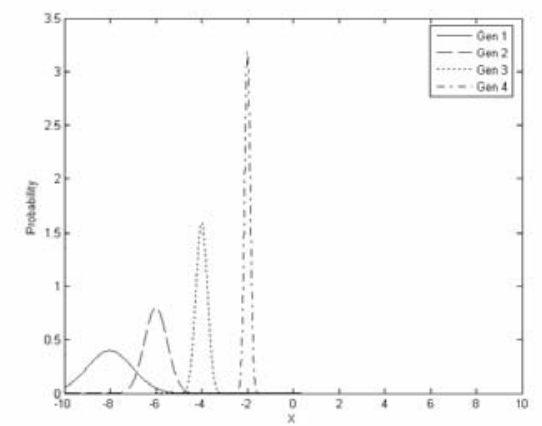

Fig. 2. A demo of the dynamics of the EDA on the unimodal problem
With the $(\mu, \lambda)$ scheme, the Gaussian model to be built in the $i^{\text {th }}$ generation is completely determined by selected individuals in the $\mathrm{i}^{\text {th }}$ population sampled from the Gaussian model built in the $(\mathrm{i}-1)^{\mathrm{th}}$ generation. As a result, there is a simple recursive relationship among the models in different generations. Another important feature is that the selected individuals in any generation are restricted within a continuous range $[-\alpha, \alpha]$ as shown in Figure 1 with the value of $\alpha$ depending on the model parameters as well as the selection pressure $\tau(0<\tau<1)$. This is because Truncation Selection applies a deterministic fitness threshold and only individuals strictly better than it could be chosen.

The specific value of $\alpha$ in the $(i+1)^{\text {th }}$ generation can be calculated by solving the following equation:

$$
\int_{-a}^{a} \frac{1}{\sqrt{2 \pi \delta_{i}^{2}}} e^{-\frac{\left(x-\mu_{i}\right)^{2}}{2 \delta_{i}^{2}}} d x=\tau
$$

The meaning of Eq. 1 is that the cumulative density of the Gaussian distribution within $[-\alpha, \alpha]$ should be equal to $\tau$, which is the portion of individuals to be selected. Although it may be difficult to come up with an analytical solution to Eq.1, it is easy to see that the value of the left hand side increases from zero monotonically as $\alpha \rightarrow+\infty$, which makes it possible to use a simple line searching method to find the appropriate $\alpha$ value at a desired accuracy level.

Once the value of $\alpha$ is available, the mean parameter $\mu_{i+1}$ and standard deviation parameter $\delta_{i+1}$ are determined by the statistics of the individuals within $[-\alpha, \alpha]$ :

$$
\begin{gathered}
\mu_{i+1}=\frac{1}{\tau} \cdot \int_{-a}^{a} x \cdot \frac{1}{\sqrt{2 \pi \delta_{i}^{2}}} e^{-\frac{\left(x-\mu_{i}\right)^{2}}{2 \delta_{i}^{2}}} d x \\
\delta_{i+1}^{2}=\frac{1}{\tau} \cdot \int_{-a}^{a}\left(x-\mu_{i+1}\right)^{2} \cdot \frac{1}{\sqrt{2 \pi \delta_{i}^{2}}} e^{-\frac{\left(x-\mu_{i}\right)^{2}}{2 \delta_{i}^{2}}} d x
\end{gathered}
$$

Now we have enough tools to describe the EDA's behavior on unimodal problems. It should be pointed out that, in the above three equations, there is no utilization of any specific information of test functions. This shows that the convergence behavior of the EDA in this situation is independent of the problems except their general structure (i.e., strictly unimodal, symmetric with regard to the origin).

In the next, some case studies are to be conducted to formally investigate the dynamics of the EDA with regard to various algorithm factors. Figures $3 \& 4$ shows the standard deviations and the mean values of the EDA with $\mu_{0}=-20$ and $\tau=0.3$ where the three lines in each plot represent results with $\delta_{0}=0.1,1.0$ and 5.0 respectively. A few interesting things that can be observed from the above case study are summarized as below:

- When the mean of the Gaussian model was far from the global optimum (i.e., several standard deviations away from the origin), the trajectories of the standard deviation values were approximately logarithmically linear, which means that the standard deviations of the Gaussian model reduced exponentially. 
- The gradient of the trajectory was independent of $\delta_{0}$ (i.e., all standard deviation trajectories were parallel to each other).

- The EDA could still get stuck on unimodal problems even with an infinite population, especially with small initial standard deviations as shown by the flat curves in Figure 4.

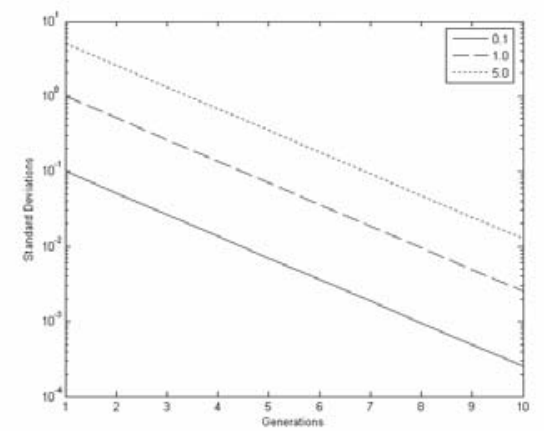

Fig. 3. The trajectories of the standard deviation parameter of the Gaussian model on unimodal problems with different initial $\delta_{0}$ values

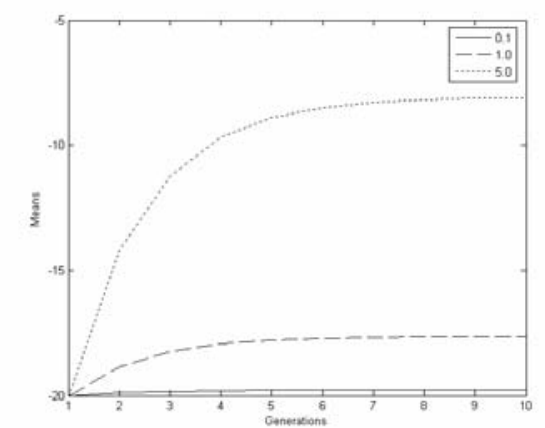

Fig. 4. The trajectories of the mean parameter of the Gaussian model on unimodal problems with different initial $\delta_{0}$ values

In order to demonstrate the influence of the selection pressure on the dynamics of the EDA, the standard deviations and the mean values of the EDA with $\mu_{0}=-20$ and $\delta_{0}=1.0$ are shown in Figures $5 \& 6$ where the three lines represent results with selection pressure $\tau=0.1,0.3$ and 0.5 respectively. It is clear that as the selection pressure increased, the convergence speed of the EDA also increased accordingly (i.e., the slope of the trajectory of the standard deviation values became steeper). Again, no satisfactory performance could be achieved as the EDA quickly got stuck somewhere far from the optimum.

Note that with a weak selection pressure (i.e., $\tau=0.5$ ), the EDA converged relatively slowly but only made limited progress before getting stuck. This is because the larger the portion of individuals to be selected, the smaller the distance between $\mu_{\mathrm{i}}$ and $\mu_{\mathrm{i}+1}$. In the extreme situation where $\tau=1.0$, the EDA will simply keep building a new model based on the population generated from the previous model, which will result in a stationary statistical model and no progress could be expected. Certainly, in practical situations where the population sizes are limited, the EDA is expected to present some kind of random walking behaviour.

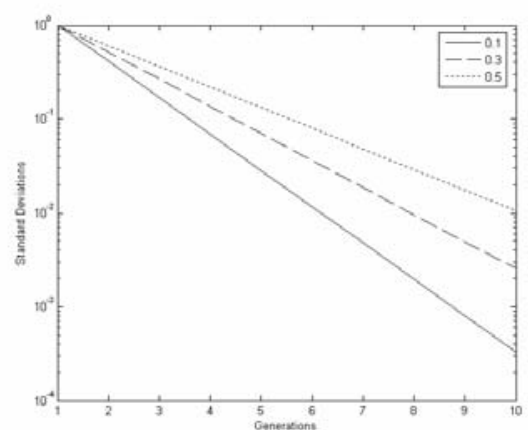

Fig. 5. The trajectories of the standard deviation parameter of the Gaussian model on unimodal problems with different selection pressures

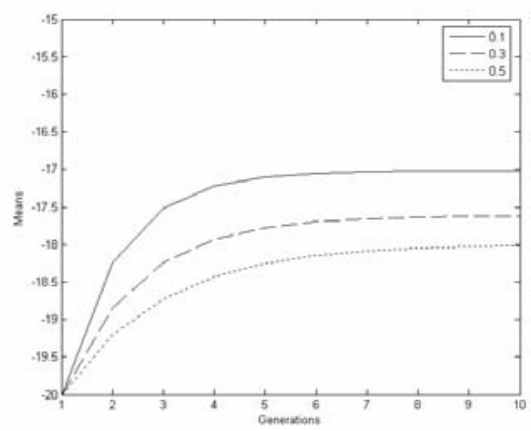

Fig. 6. The trajectories of the mean parameter of the Gaussian model on unimodal problems with different selection pressures

In the above two case studies, the EDA was never able to converge to the global optimum and, as suggested by our previous research[12], it is necessary to explicitly maintain the population diversity to prevent the EDA from converging too quickly. A simple approach is to introduce a new algorithm parameter $\gamma$, which is used to amplify the original standard deviation parameter (i.e., $\delta_{\mathrm{i}}^{\prime}=\gamma \cdot \delta_{\mathrm{i}}$ ). Certainly, for $\gamma=1.0$, the EDA will be kept unchanged. Figures $7 \& 8$ shows the standard deviations and the mean values of the EDA with $\mu_{0}=-20, \delta_{0}=1.0$ and $\tau=0.3$ where the three lines in each plot represent results with $\gamma=1.0,1.5$ and 2.0 respectively. It is evident that this new parameter could dramatically change the dynamics of the EDA. With $\gamma>1.0$, the standard deviations were often orders of magnitude larger than in the original EDA. In the meantime, the mean of the Gaussian model could also consistently move towards the global optimum with $\gamma=2.0$, showing some significantly improved performance.

However, it does not necessarily mean that such large $\gamma$ values should be used in practice where the population size is limited. The issue is that the standard deviation may increase during evolution and become quite large, which makes the EDA close to doing random search. Since the distance between the current model and the global optimum is typically not known in optimization, having the standard deviation increase or decrease may all seem to be dangerous. As a result, a good strategy is to keep the current standard deviation value unchanged. Recall that, when the EDA is far from the global optimum, its convergence trajectory is only influenced by $\tau$ and is independent of the initial standard deviation. So, for different $\tau$ values, there are corresponding 
optimum $\gamma$ values (i.e., $\gamma^{*}=\delta_{\mathrm{i}} / \delta_{\mathrm{i}+1}$ ). For example, the $\gamma^{*}$ values for $\tau=0.1,0.3 \& 0.5$ are approximately $2.4315,1.9443$ and 1.6589 respectively.

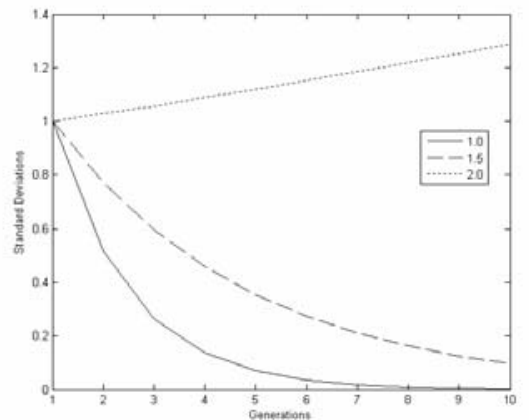

Fig. 7. The trajectories of the standard deviation parameter of the Gaussian model on unimodal problems with diversity maintenance

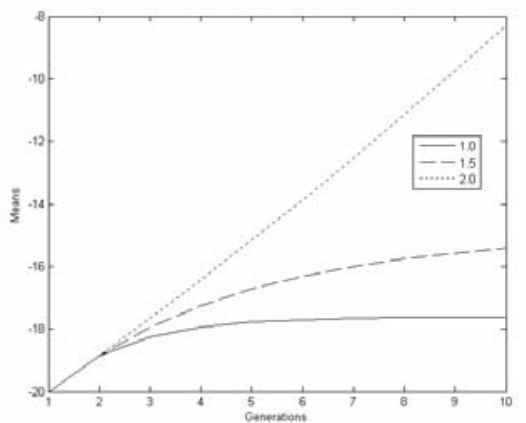

Fig. 8. The trajectories of the mean parameter of the Gaussian model on unimodal problems with diversity maintenance

In order to demonstrate the effect of the optimum $\gamma$ values, we investigated both empirically and theoretically the EDA with $\gamma^{*}=1.9943$ in accordance with $\tau=0.3$. The EDA started with $\mu_{0}=-20$ and $\delta_{0}=1.0$ and, for empirical studies, the population size was 5000 and results were averaged over 25 independent trials.

Figure 9 shows that the standard deviation of the EDA was almost unchanged within the first 16 generations due to the $\gamma^{*}$ value in use. After that, when the EDA was quite close to the global optimum as shown in Figure 10, it started converging very quickly and changed its behavior to local searching. This is because when it is far from the global optimum, the problem could be regarded as being monotonous and its behavior is approximately consistent. However, the gradient of the convergence trajectory of the EDA gets significantly larger when it is close to the global optimum and the original $\gamma^{*}$ value is no longer large enough to keep the standard deviation unchanged. This phenomenon could be roughly explained by the fact that when the Gaussian model is very close to the optimum, selected individuals tend to distribute within a much smaller range than when the Gaussian model is far from the optimum.

In fact, this property is very useful as it could adapt the EDA's behavior depending on its current situation (i.e., this is an important advantage over a hill-climbing algorithm with fixed step size). At last, the empirical results marked by '+' had a good match against the theoretical analysis, which verified the soundness of our methods.

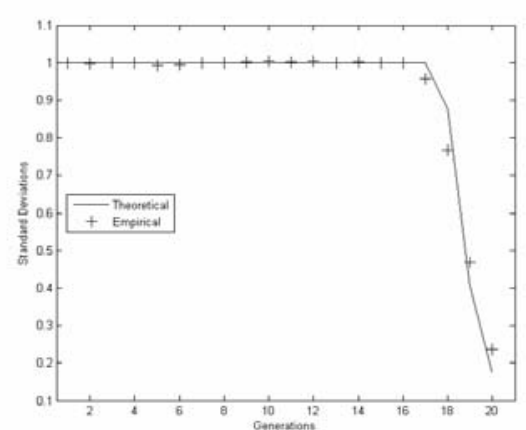

Fig. 9. The trajectory of the standard deviation parameter of the Gaussian model on unimodal problems with diversity maintenance $\left(\gamma^{*}=1.9443, \tau=0.3\right)$

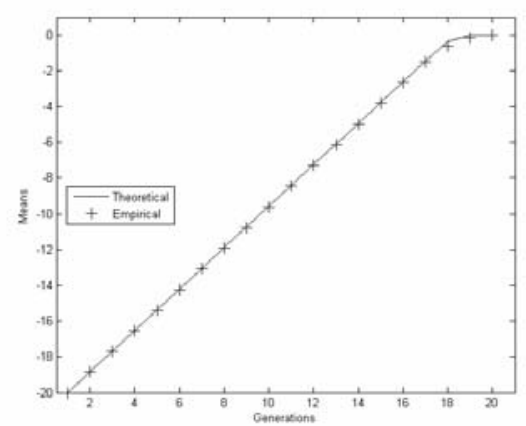

Fig. 10. The trajectory of the mean parameter of the Gaussian model on unimodal problems with diversity maintenance $\left(\gamma^{*}=1.9443, \tau=0.3\right)$

\section{MODELLING MULTIMODAL PROBLEMS}

In this section, we will apply the modelling techniques in a more general way. Firstly, the problems to be solved have a global optimum and a local optimum. Secondly, in addition to the relatively simple $(\mu, \lambda)$ scheme, the $(\mu+\lambda)$ scheme is also considered, which choose the best individuals from the union of both old and new populations.

For the following analysis and experiments, test problems (i.e., to be maximized) are constructed by two Gaussian functions due to their smoothness and symmetric shape (i.e., this has nothing to do with the Gaussian model of the EDA). The fitness value of $x$ is determined by the Gaussian function that gives it the larger value. Each Gaussian function is governed by its $\mu_{\mathrm{j}}$ and $\delta_{\mathrm{j}}$ while $\omega_{\mathrm{j}}$ is used to adjust its height.

$$
\begin{gathered}
F(x)=\max \left\{G_{1}(x), G_{2}(x)\right\} \\
\text { with } \\
G_{j}(x)=\omega_{j} \cdot \frac{1}{\sqrt{2 \pi \delta_{j}^{2}}} e^{-\frac{\left(x-\mu_{j}\right)^{2}}{2 \delta_{j}^{2}}} \quad \omega_{j}>0
\end{gathered}
$$

Two examples are shown in Figures $11 \& 12$ with different parameter values. When there are two or more peaks in the problem, it is not always sufficient to describe the range of selected individuals by a single interval because they may come from different peaks. In Figure 11, when the worst fitness value (selection threshold) indicated by line $\mathrm{A}$ is greater than the value of the local optimum at $\mathrm{x}=0.2$, all selected individuals will come from the peak corresponding 
to the global optimum at $\mathrm{x}=-0.3$ and bounded within $\left[\mathrm{x}_{1}, \mathrm{x}_{2}\right]$, which is similar to the unimodal case. On the other hand, when the threshold is lower, as indicated by line $B$, the selected individuals will be from both peaks and two intervals $\left[\mathrm{x}_{1}, \mathrm{x}_{2}\right]$ and $\left[\mathrm{x}_{3}, \mathrm{x}_{4}\right]$ are required. Furthermore, when the two peaks are very close, it is possible that only one interval is needed, as shown in Figure 12.

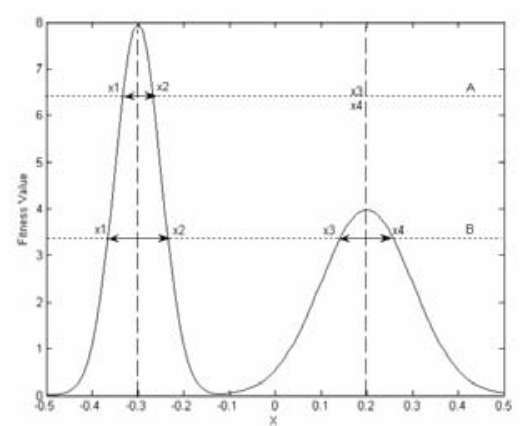

Fig. 11. A multimodal problem with two separate optima

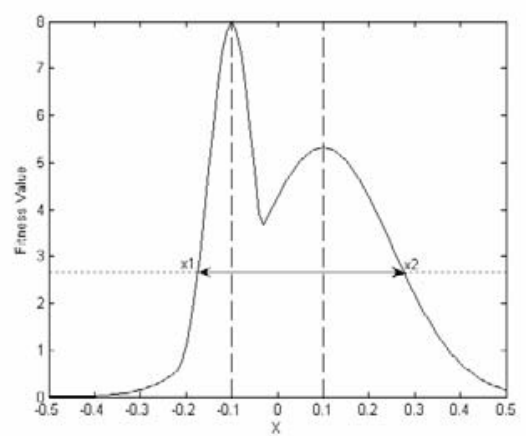

Fig. 12. A multimodal problem with two close optima

Similarly, the initial population is sampled from a Gaussian $\left(\mu_{0}, \delta_{0}\right)$. In general, with the $(\mu, \lambda)$ scheme, the two intervals at the $(i+1)^{\text {th }}$ generation represented by $\left[\mathrm{x}_{1}, \mathrm{x}_{2}\right]$ and $\left[\mathrm{x}_{3}, \mathrm{x}_{4}\right]$ could be calculated by solving the following equation (i.e., solved by line searching in practice):

$$
\begin{gathered}
\int_{x_{1}}^{x_{2}} P(x, i) d x+\int_{x_{3}}^{x_{4}} P(x, i) d x=\tau \\
\text { with } \\
P(x, i)=\frac{1}{\sqrt{2 \pi \delta_{i}^{2}}} e^{-\frac{\left(x-\mu_{i}\right)^{2}}{2 \delta_{i}^{2}}} \\
G_{1}\left(x_{1}\right)=G_{1}\left(x_{2}\right)=G_{2}\left(x_{3}\right)=G_{2}\left(x_{4}\right)
\end{gathered}
$$

In situations like the threshold $\mathrm{A}$ in Figure 11, the additional condition will reduce to $\mathrm{G}_{1}\left(\mathrm{x}_{1}\right)=\mathrm{G}_{1}\left(\mathrm{x}_{2}\right)$, assuming $\mathrm{G}_{1}(\mathrm{x})$ corresponds to the global optimum. Furthermore, in situations like Figure 12, the two intervals $\left[\mathrm{x}_{1}, \mathrm{x}_{2}\right]$ and $\left[\mathrm{x}_{3}, \mathrm{x}_{4}\right]$ satisfying the additional condition may overlap with each other. Consequently, the two intervals must be merged together. In both cases $\mathrm{x}_{3}$ and $\mathrm{x}_{4}$ are set to some identical values, representing an empty interval.
Once the intervals are determined, the new model parameters are calculated in a similar manner as in the unimodal case (i.e., $\Omega=\left[x_{1}, x_{2}\right] \cup\left[x_{3}, x_{4}\right]$ ):

$$
\begin{gathered}
\mu_{i+1}=\frac{1}{\tau} \cdot \int_{\Omega} x \cdot P(x, i) d x \\
\delta_{i+1}^{2}=\frac{1}{\tau} \cdot \int_{\Omega}\left(x-\mu_{i+1}\right)^{2} \cdot P(x, i) d x
\end{gathered}
$$

The major difference between the $(\mu, \lambda)$ scheme and the $(\mu+\lambda)$ scheme is that the latter may keep old individuals of high quality to the next generation. That is to say, the $i^{\text {th }}$ population is not solely sampled from the statistical model built in the (i-1) ${ }^{\text {th }}$ generation. Instead, it can be decomposed into a subset of individuals generated by the initial Gaussian model (i.e., the initial population) and i-1 subsets of individuals generated from the previous i-1 models respectively. Following this rule, it is easy to work out the new equations with the similar meaning as Eqs. 5-7:

$$
\begin{gathered}
\sum_{k=0}^{i}\left[\int_{x_{1}}^{x_{2}} P(x, k) d x+\int_{x_{3}}^{x_{4}} P(x, k) d x\right]=\tau \\
\mu_{i+1}=\frac{1}{\tau} \cdot\left[\sum_{k=0}^{i} \int_{\Omega} x \cdot P(x, k) d x\right] \\
\delta_{i+1}^{2}=\frac{1}{\tau} \cdot\left[\sum_{k=0}^{i} \int_{\Omega}\left(x-\mu_{i+1}\right)^{2} \cdot P(x, k) d x\right]
\end{gathered}
$$

A test problem was constructed according to Eq. 4 with the global optimum created by a Gaussian $(-0.5,0.05)$ as well as a local optimum created by a Gaussian $(0.5,0.2)$, which was scaled up by a factor of 3.5 to make it a competitive optimum (i.e., a large basin as well as $87.5 \%$ of the fitness value of the global optimum).

The initial Gaussian model was set as $\mu_{0}=0$ and $\delta_{0}=0.5$. Since the initial Gaussian model was centred in the middle between the two optima, it is likely that, at least at the beginning of evolution, quite a large portion of selected individuals might come from the peak corresponding to the local optimum due to its significantly larger basin size. From this point of view, this problem is deceptive and it would be interesting to see how the EDA could handle this difficulty with the two selection schemes.

The dynamics of the EDA with the $(\mu+\lambda)$ scheme is shown in Figures $13 \& 14$ (i.e., $\tau=0.3$ ). Compared to the monotonous behaviour observed in the unimodal case, the behaviour of the EDA is much more complicated on multimodal problems. For example, in the first five generations, the standard deviation values were shrinking and, in the meantime, the mean of the Gaussian was moving towards the local optimum.

This indicates that the EDA was being misled by the large portion of selected individuals from the local optimum. However, after that point, the EDA moved back and consistently converged towards the global optimum, correcting its previous misjudgement. 


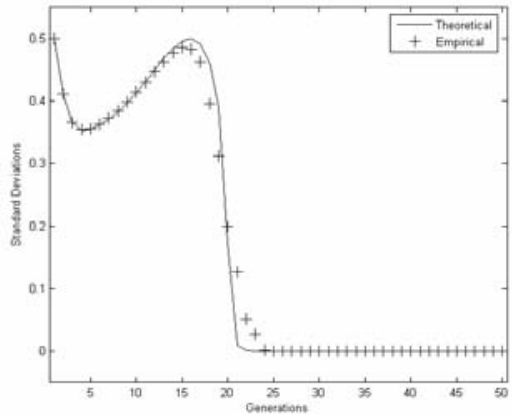

Fig. 13. The trajectory of the standard deviation parameter of the Gaussian model on the multimodal test problem with the $(\mu+\lambda)$ scheme

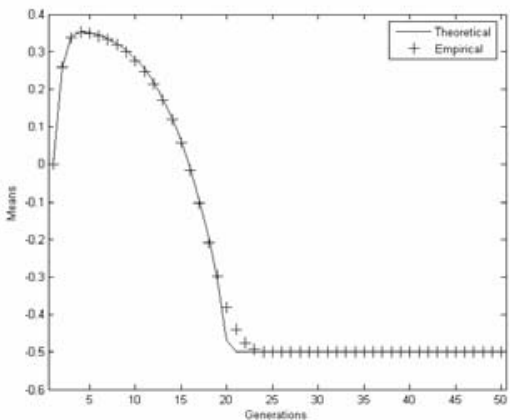

Fig. 14. The trajectory of the mean parameter of the Gaussian model on the multimodal test problem with the $(\mu+\lambda)$ scheme

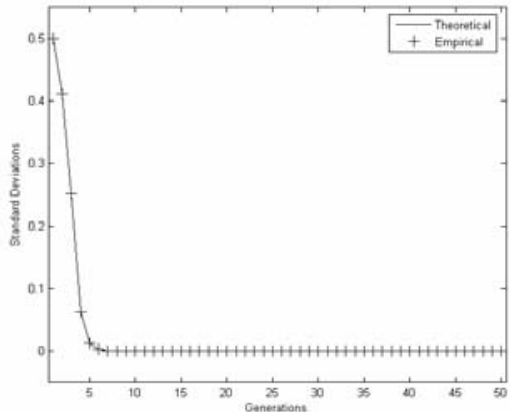

Fig. 15. The trajectory of the standard deviation parameter of the Gaussian model on the multimodal problem with the $(\mu, \lambda)$ scheme

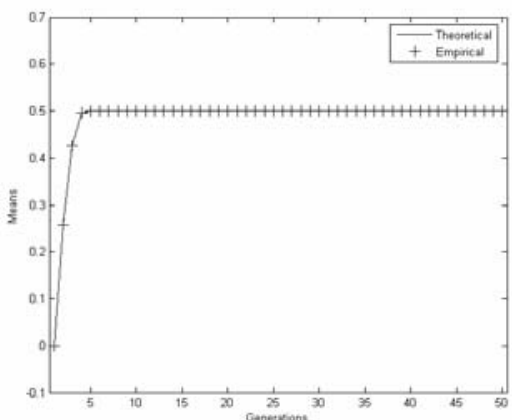

Fig. 16. The trajectory of the mean parameter of the Gaussian model on the multimodal problem with the $(\mu, \lambda)$ scheme
It should be pointed out that the EDA with the $(\mu+\lambda)$ scheme will never get stuck at the local optimum with an infinitely large population provided that every point in the search space has the chance to be sampled based on the statistical model in use, even with small probabilities. This is because the $(\mu+\lambda)$ scheme could always memorize good individuals that have been found and those individuals from the basin corresponding to the global optimum (i.e., it is guaranteed that some of them would exist in the initial population) could not only prevent the EDA from converging to the local optimum infinitely but also gradually update the model towards the global optimum.

By contrast, there could be a quite different story with the $(\mu, \lambda)$ scheme in which all old individuals are discarded. As long as individuals from the local optimum start dominating the population, the model will be updated towards the local optimum and the chance of getting new individuals from the global optimum will decrease, further reducing its influence in the population. In Figure 14, the standard deviation quickly dropped to nearly zero after five generations while, as shown in Figure 15, the mean got stuck at 0.5 where the local optimum was located. Again, in both cases, empirical results (i.e., population size $=10,000$ and 25 independent trials) had a good match against the theoretical analysis.

\section{CONCLUSIONS}

A mathematical modelling technique is proposed under the assumption of infinite population size to investigate the dynamics of a continuous EDA with Truncation Selection and Gaussian models. The importance of this technique is that it provides a principled method to analyse the behaviour of the EDA under the influence of different algorithm / problem factors.

In the most general situation, the first step is to identify the area where the currently best individuals are distributed. Typically, this area is specified by the contours of the fitness function. With the $(\mu, \lambda)$ scheme, this area represented by $\Omega$ should satisfy the following condition where $\mathrm{P}(\mathrm{X}, \mu, \Sigma)$ is the density function of multivariate Gaussian distributions:

$$
\int_{\Omega} P\left(X, \mu_{i}, \Sigma_{i}\right) d X=\tau
$$

The mean value of the new Gaussian model in the jth dimension could then be calculated by:

$$
\mu_{i+1, j}=\frac{1}{\tau} \int_{\Omega} X_{j} P\left(X, \mu_{i}, \sum_{i}\right) d X
$$

The standard deviation of the new Gaussian model in the jth dimension is given by:

$$
\delta_{i+1, j}^{2}=\frac{1}{\tau} \int_{\Omega}\left(X_{j}-\mu_{i+1, j}\right)^{2} P\left(X, \mu_{i}, \sum_{i}\right) d X
$$

Finally, the covariance between the jth and the kth variables is given by:

$$
\delta_{i+1, j k}^{2}=\frac{1}{\tau} \int_{\Omega}\left(X_{j}-\mu_{i+1, j}\right)\left(X_{k}-\mu_{i+1, k}\right) P\left(X, \mu_{i}, \sum_{i}\right) d X
$$


In the case studies, this technique is applied to $1 \mathrm{D}$ unimodal and multimodal problems and some interesting properties of the EDA have been found some of which are unknown in previous research.

For 1D unimodal problems with the $(\mu, \lambda)$ scheme:

- The dynamics of the EDA is independent of the test function except of its general shape, which is also true for the $(\mu+\lambda)$ scheme.

- When starting far away from the global optimum, the EDA may get stuck.

- Given a certain selection pressure, there is a unique $\gamma^{*}$ that could help the EDA achieve desirable performance by maintaining the diversity.

For 1D multimodal problems:

- The EDA could get stuck with the $(\mu, \lambda)$ scheme.

- The EDA will never get stuck with the $(\mu+\lambda)$ scheme, which is also true for unimodal problems for obvious reasons.

- The above conclusions also hold for high dimensional problems.

Furthermore, the proposed method could be easily adapted to model other aspects of the evolution such as the portion of individuals on each optimum or the mean fitness value of the population. As to higher dimensional problems, some preliminary work has been successfully conducted on 2D problems with and without dependences among parameters. However, a major challenge may come from specifying the boundaries of selected individuals and calculating multiple integrals over several variables. As a result, it seems that some further assumptions may be required to keep the complexity of such theoretical analysis at a reasonable level.

At last, we have also noticed the connection between the theoretical analysis of EDAs and the existing work on Evolution Strategies [3], which may help these two areas benefit from each other.

\section{ACKNOWLEDGMENT}

This work was supported by an Australian Postgraduate Award granted to Bo Yuan.

\section{REFERENCES}

[1] T. Back, D. B. Fogel, and Z. Michalewicz, "Handbook of Evolutionary Computation," IOP Publishing Ltd and Oxford University Press, 1997.

[2] A. Berny, "Selection and Reinforcement Learning for Combinatorial Optimization," In Parallel Problem Solving from Nature VI, 2000.

[3] H.-G. Beyer, The theory of evolution strategies: Springer, 2001.

[4] C. González, J. A. Lozano, and P. Larrañaga, "Analyzing the PBIL algorithm by means of discrete dynamical systems," Complex Systems, vol. 12 (4), pp. 465-479, 2000.

[5] C. González, J. A. Lozano, and P. Larrañaga, "Mathematical Modelling of UMDAc algorithm with tournament selection. Behaviour on linear and quadratic functions," International Journal of Approximate Reasoning, vol. 31, pp. 313-340, 2000.

[6] J. Grahl, S. Minner, and F. Rothlauf, "Behaviour of UMDAc with Truncation Selection on Monotonous Functions," In Congress on Evolutionary Computation 2005, 2005.

[7] M. Hohfeld and G. Rudolph, "Towards a Theory of PopulationBased Incremental Learning," In IEEE International Conference on Evolutionary Computation (ICEC '97), 1997.

[8] P. Larrañaga and J. A. Lozano, "Estimation of Distribution Algorithms: A New Tool for Evolutionary Computation," Kluwer Academic Publishers, 2001.

[9] H. Mühlenbein, "The Equation for Response to Selection and Its Use for Prediction," Evolutionary Computation, vol. 5 (3), pp. 303 - 346, 1997.

[10] H. Mühlenbein and T. Mahnig, "Convergence Theory and Applications of the Factorized Distribution Algorithm," Journal of Computing and Information Technology, vol. 7 (1), pp. 19-32, 1999.

[11] J. L. Shapiro, "Scaling of Probability-based Optimization Algorithms," In Advances in Neural Information Processing Systems 15 (NIPS2002), 2003.

[12] B. Yuan and M. Gallagher, "On the Importance of Diversity Maintenance in Estimation of Distribution Algorithms," In the 2005 Genetic and Evolutionary Computation Conference, 2005.

[13] Q. Zhang and H. Mühlenbein, "On the convergence of a class of estimation of distribution algorithms," IEEE Transactions on Evolutionary Computation, vol. 8 (2), pp. 127- 136, 2004. 